Sociologie et sociétés

\title{
Liste des personnes ayant préparé des évaluations de manuscrits pour les numros de Sociologies et sociétés de 1994-1995 et 1995-1996
}

\section{List of Manuscript Evaluators for Sociologie et sociétés in 1994-1995 and 1995-1996}

Volume 28, numéro 2, automne 1996

Technologies médicales et changement de valeurs

URI : https://id.erudit.org/iderudit/001446ar

DOI : https://doi.org/10.7202/001446ar

Aller au sommaire du numéro

Éditeur(s)

Les Presses de l'Université de Montréal

ISSN

0038-030X (imprimé)

1492-1375 (numérique)

Découvrir la revue

Citer ce document

(1996). Liste des personnes ayant préparé des évaluations de manuscrits pour les numros de Sociologies et sociétés de 1994-1995 et 1995-1996. Sociologie et sociétés, 28(2), 199-202. https://doi.org/10.7202/001446ar 


\section{Liste des personnes ayant préparé des évaluations de manuscrits pour Sociologie et sociétés*}

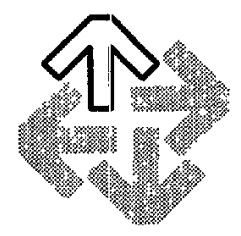

La direction tient à exprimer sa profonde reconnaissance aux collègues qui ont bien voulu accepter d'évaluer un ou plusieurs manuscrits soumis à la revue, en vue des numéros de 19941995 et 1995-1996.

1994-1.995

M. Pierre ANSART, Département de sociologie, Université de Paris VII M. Michel AUDET, Faculté des sciences de l'administration, Université Laval M. Claude BARITEAU, Département d'anthropologie, Université Laval M. Philippe BATAILLE, Département de sociologie, Université de Montréal M. Rob BEAMISH, School of Health and Physical Education, Queen's University M André J. BÉLANCER, Département de science politique, Université de Montréal M. Paul R. BÉLANGER, Département de sociologie, Université du Québec à Montréal M. Gérard BOISMENU, Département de science politique, Université de Montréal M. Gary CALDWELL, sociologue indépendant, Martinville, Québec M. Maurice CHARLAND, Département de communication, Université Concordia M. Olivier CLAIN, Département de sociologie, Université Laval M. Jay COAKLEY, Department of Sociology, University of Colorado at Colorado Springs M. Patrick COHENDET, Bureau d'économie théorique et appliquée, Université Louis-Pasteur, Strasbourg

Mme Denise COUTURE, Département de sociologie, Université de Montréal M. Pierre DANDURAND, Département de sociologie, Université de Montréal Mme Monique de SAINT-MARTIN, Centre de sociologie de l'éducation et de la culture, École des hautes études en sciences sociales, Paris

Mme Francine DESCARRIES, Département de sociologie, Université du Québec à Montréal M. Daniel DOS SANTOS, Département de criminologie, Université d'Ottawa M. Jacques DUFRESNE, L'AGORA, Ayer's Cliff, Québec Mme Brigitte DUMAS, Département de sociologie, Université de Montréal M. Michel FREITAG, Département de sociologie, Université du Québec à Montréal

* Quelques-unes de ces personnes ont évalué plus d'un manuscrit. 
M. Gabriel GAGNON, Département de sociologie, Université de Montréal

M. Jean-Claude GARDIN, Direction d'études «Sémiologie et Informatique», École des hautes études en sciences sociales, Paris

M. Jacques T. GODBOUT, Institut national de la recherche scientifique-Urbanisation, Montréal

M. Jacques GRAND-MAISON, Évêché de Saint-Jérôme, Québec

Mme M. Ann HALL, Department of Physical Education and Sport Studies, University of Alberta

M. John HORNE, The Scottish Centre for Physical Education Movement and Leisure Studies, Institute of Education, Heriot-Watt University

M. Roch HURTUBISE, Département de service social, Université de Sherbrooke

M. Alan G. INGHAM, Department of Physical Education, Health and Sport Studies, School of Education and Allied Professions, Miami University

M. Simon LANGLOIS, Département de sociologie, Université Laval

Mme Évelyne LAPIERRE-ADAMCYK, Département de démographie, Université de Montréal

Mme Céline LE BOURDAIS, Institut national de la recherche scientifique-Urbanisation, Montréal

M. Raymond LEMIEUX, Groupe de recherche en sciences de la religion, Université Laval

M. Jocelyn LÉTOURNEAU, Département d'histoire, Université Laval

Mme Catherine LOUVEAU, Laboratoire de sociologie du sport, Institut national du sport et de l'éducation physique, Paris

M. John J. MacALOON, Master of Arts Program in the Social Sciences, The University of Chicago

Mme Chantal MAILLÉ, Institut Simone-de-Beauvoir, Université Concordia

M. Danilo MARTUCCELLI, Département de sociologie, Université de Bordeaux II

Mme Marie McANDREW, Département d'études en éducation et d'administration de l'éducation, Université de Montréal

M. Jim McKAY, Department of Anthropology and Sociology, The University of Queensland

M. Robert A. MECHIKOFF, Department of Physical Education, San Diego State University

M. Michael A. MESSNER, Department of Sociology, University of Southern California

M. Jorge NIOSI, Département de sociologie, Université du Québec à Montréal

M. Alain NOËL, Département de science politique, Université de Montréal

Mme Catherine PARADEISE, Département Économie et Gestion, École normale supérieure de Cachan

M. Victor PICHÉ, Département de démographie, Université de Montréal

M. Jean PICHETTE, Groupe interuniversitaire d'étude de la post-modernité, Département de sociologie, Université du Québec à Montréal

M. Marcel RAFIE, Département de sociologie, Université du Québec à Montréal

Mme Nicole RAMOGNINO, Département de sociologie, Université de Provence (AixMarseille I)

M. Gilbert RENAUD, École de service social, Université de Montréal

M. Philip RESNICK, Department of Political Science, The University of British Columbia

M. François RICARD, Département de langue et littérature françaises, Université McGill

M. Jan RINTALA, Department of Physical Education, Northern Illinois University

M. Guy ROCHER, Centre de recherche en droit public, Université de Montréal

M. Paul SABOURIN, Département de sociologie, Université de Montréal

M. Daniel SALÉE, School of Community and Public Affairs, Université Concordia

M. Stephen SCHECTER, Département de sociologie, Université du Québec à Montréal

M. Charles SUAUD, Département de sociologie, Université de Nantes

M. J. Yvon THÉRIAULT, Département de sociologie, Université d'Ottawa

M. Alan TOMLINSON, Chelsea School of Physical Education, Sports Science, Dance and Leisure, University of Brighton 
M. André TURMEL, Département de sociologie, Université Laval M. Jean-Guy VAILLANCOURT, Département de sociologie, Université de Montréal M. Yves VAILLANCOURT, Département de travail social, Université du Québec à Montréal M. Robert VANDYCKE, Département de sociologie, Université de Montréal Mme Denise VEILLETTE, Département de sociologie, Université Laval M. Kevin YOUNG, Department of Sociology, The University of Calgary Mme Anne-Marie WASER, École des hautes études en sciences sociales, Paris Mme Deena WHITE, Département de sociologie, Université de Montréal

1995-1996

M. Ronald BABIN, Département de sociologie, Université de Moncton M. Claude BARITEAU, Département d'anthropologie, Université Laval Mme Bernadette BAWIN-LEGROS, Département de sociologie, Université de Liège M. Roderic BEAUJOT, Department of Sociology, The University of Western Ontario M. Paul BERNARD, Département de sociologie, Université de Montréal

M. Jacques BRAZEAU, Département de sociologie, Université de Montréal

M. Jean Michel BERTHELOT, Centre de recherches sociologiques, Université de ToulouseLe Mirail

M. Marc BESSIN, Groupe de recherche et d'analyse du social et de la sociabilité, CNRSIRESCO, Paris

Mme Bernadette BLANC, Institut d'urbanisme, Université de Montréal

M. Gérard BOISMENU, Département de science politique, Université de Montréal

M. Pierre BOUVIER, Département de sociologie, Centre national de la recherche scientifique, Laboratoire de sociologie du changement institutionnel, IRESCO, Paris

Mme Johanne CHARBONNEAU, Institut national de la recherche scientifique-Urbanisation, Montréal

Mme Adèle CHENÉ, Département d'études en éducation et d'administration de l'éducation, Université de Montréal

M. Pierre CHIFFLET, U.F.R. - Activités physiques et sportives, Université Joseph-Fourier (Grenoble I)

M. Jean-Paul CLÉMENT, Centre de recherches interdisciplinaires-STAPS, Université des sciences humaines de Strasbourg

M. François COLBERT, Chaire de gestion des arts, École des hautes études commerciales, Montréal

Mme Christime CORBEIL, Département de travail social, Université du Québec à Montréal

Mme Denise COUTURE, Département de sociologie, Université de Montréal

Mme Lucie France DAGENAIS, Direction de la recherche, Commission des droits de la personne du Québec, Montréal

Mme Francine DANSEREAU, Institut national de la recherche scientifique-Urbanisation, Montréal

Mme Maria DE KONINCK, Département de médecine sociale et préventive, Université Laval M. Alain DESROSIÈRES, Centre de recherche en économie et statistique - INSEE, Malakoff Mme Lorraine DUCHESNE, Institut national d'études démographiques, Paris

Mme Brigitte DUMAS, Département de sociologie, Université de Montréal

M. Pascal DURET, Laboratoire de sociologie, Institut national du sport et de l'éducation physique, Paris

M. Patrick FESTY, Institut national d'études démographiques, Paris

M. Guy FRÉCHET, Ministère de la Santé et des Services sociaux, Gouvernement du Québec

Mme Micheline FRENETTE, Département de communication, Université de Montréal

Mme Danielle GAUVREAU, Département de sociologie et d'anthropologie, Université Concordia

Mme Corinne GENDRON, Trivert Inc., Montréal 
Mme Annick GERMAIN, Institut national de la recherche scientifique-Urbanisation, Montréal

M. Jacques T. GODBOUT, Institut national de la recherche scientifique-Urbanisation, Montréal

Mme Anne-Marie GUILLEMARD, Centre d'études des mouvements sociaux, Paris

M. Pierre HAMEL, Institut d'urbanisme, Université de Montréal

M. Nicolas HERPIN, Institut national de la statistique et des études économiques, Paris

M. Jacques JENNY, Centre national de la recherche scientifique, Institut de recherche sur les sociétés contemporaines, GEDISST, Paris

M. Alain JOYAL, Département des sciences sociales, collège Lionel-Groulx, Sainte-Thérèse, Québec

Mme Danièle KERGOAT, Institut de recherche sur les sociétés contemporaines, CNRSGEDISST, Paris

M. Michel KOKOREFF, Institut de recherche et d'information socio-économique, Université de Paris Dauphine

Mme Marie-France LABRECQUE, Département d'anthropologie, Université Laval

M. Simon LANGLOIS, Département de sociologie, Université Laval

Mme Évelyne LAPIERRE-ADAMCYK, Département de démographie, Université de Montréal

M. Serge LAROSE, Département de sociologie, Université du Québec à Montréal et Cégep de Sant-Hyacinthe

M. Jacques LAZURE, Département de sociologie, Université du Québec à Montréal

Mme Solange LEFEBVRE, Faculté de théologie, Université de Montréal

M. Didier LE GALL, Département de sociologie, Université de Caen Basse-Normandie

Mme Éva LELIÈVRE, Institut national d'études démographiques, Paris

M. Henri LERIDON, Institut national d'études démographiques, Paris

M. Marc LESAGE, Département de sociologie, Collège universitaire Glendon, Université York

M. Ronny J. LESTHAEGHE, Centrum voor Sociologie, Vrije Universiteit Brussel

M. Benoît LÉVESQUE, Département de sociologie, Université du Québec à Montréal

M. Henri LUSTIGER-THALER, School of Social Sciences and Human Services, Ramapo College of New Jersey

Mme Jacqueline MASSÉ, Département de sociologie, Université de Montréal

M. Gérard MAUGER, CNRS, Centre de sociologie urbaine, Paris

M. Robert MAYER, École de service social, Université de Montréal

M. Guy MÉNARD, Département des sciences religieuses, Université du Québec à Montréal

M. Alain NOËL, Département de science politique, Université de Montréal

M. André PETITAT, Faculté des sciences sociales et politiques, Institut des sciences sociales et pédagogiques, Université de Lausanne

Mme Agnès PITROU, Centre national de la recherche scientifique-LEST, Aix-en-Provence

M. Luc RACINE, Département de sociologie, Université de Montréal

M. Jean-François RENÉ, Département de travail social, Université du Québec à Montréal

M. Guy ROUSTANG, CNRS, Laboratoire d'économie et de sociologie du travail, Aix-enProvence

M. Gilles ROY, Faculté de l'éducation permanente, Université de Montréal

Mme Martine SEGALEN, Centre d'ethnologie française, Musée national des arts et traditions populaires, Paris

M. Gilles SIMARD, École des sciences de la gestion, Université du Québec à Montréal

Mme Nicole TABARD, Institut national de la statistique et des études économiques, Division Études sociales, Paris

Mme Irène THERY, Observatoire sociologique du changement, Paris

M. André TURMEL, Département de sociologie, Université Laval

M. Jean-Guy VAILLANCOURT, Département de sociologie, Université de Montréal

Mme Catherine VILLENEUVE-GOKALP, Institut national d'études démographiques, Paris

M. Michel WIEVIORKA, Centre d'analyse et d'intervention sociologiques, École des hautes études en sciences sociales, Paris 